\title{
Extreme Consumers of Health Care: Patterns of Care Utilization in Patients with Multiple Chronic Conditions Admitted to a Novel Integrated Clinic
}

This article was published in the following Dove Press journal: Journal of Multidisciplinary Healthcare

\author{
Muhammad Rafiq (D) \\ George Keel (D) \\ Pamela Mazzocato (iD ${ }^{1,2}$ \\ Jonas Spaak (D) 1,3 \\ Christian Guttmann (D) ${ }^{1,4,5}$ \\ Peter Lindgren (D) ${ }^{\prime}$ \\ Carl Savage (ID)
}

'Department of Learning, Informatics, Management and Ethics, Medical Management Centre, Karolinska Institutet, Stockholm, Sweden;

${ }^{2}$ Department for Research,

Development, Education and Innovation, Södertälje Hospital, Södertälje 152 40,

Sweden; ${ }^{3}$ Department of Clinical

Sciences, Danderyd University Hospital, Karolinska Institutet, Stockholm I82 88, Sweden; ${ }^{4}$ Tieto Sweden AB, Stockholm II 5 83, Sweden; ${ }^{5}$ Nordic Artificial Intelligence Institute, Stockholm II 3 31, Sweden
Correspondence: Pamela Mazzocato Medical Management Center (MMC),

Karolinska Institutet (KI)

Tomtebodavägen 18 a, Stockholm 17177 , Sweden

Tel +46766502843

Email pamela.mazzocato@ki.se
Purpose: Patients with multiple chronic conditions (MCC) of diabetes, cardiovascular and kidney diseases; hereafter referred to as HND (heart/cardiac-, nephrology-, diabetes mellitus-) patients, are high utilizers of health care. However, the care received is often insufficiently coordinated between different specialties and health-care providers. This study aims to describe the characteristics of HND patients and to explore the initial effects of a multidisciplinary and person-centered care on total care utilization.

Patients and Methods: We conducted a sub-study of HND patients recruited in an ongoing randomized trial CareHND (NCT03362983). Descriptive statistics of patient characteristics, including diagnostic data and Charlson Comorbidity Index scores, informed a comparison of care utilization patterns between HND patient care and traditional care. Diagnostic and care utilization data were collected from a regional database. Wilcoxon signed ranked sum tests were performed to compare care utilization frequencies between the two groups.

Results: Patients included in the study were care-intensive with several diagnoses and experienced a high level of variation in care utilization and diagnoses profiles. HND patients were sicker than their counterparts in the control group. Utilization indicators were similar between the two arms. There was some indication that the HND center is beginning to perform as expected, but no results were statistically significant.

Conclusion: This study sits among many studies reporting difficulties obtaining statistically significant findings for MCC patients. However, previous research has shown that the key components of this intervention, such as integrated, multidisciplinary, inter-professional collaboration within patient-centered care have had a positive effect on health-care outcomes. More innovative methods beyond the RCT, such as machine learning should be explored to evaluate the impact of integrated care interventions on care utilization.

Keywords: integrated practice unit, health care utilization, person-centered care, multidisciplinary care

\section{Introduction}

Patients with multiple chronic conditions (MCCs) consume a large proportion of health-care expenditures. ${ }^{1-4}$ In the US, patients with MCCs often require expenditures up to seven times that of patients with a single chronic condition. ${ }^{5}$ A recent study in the US showed that patients with five or more chronic conditions, which constitute $12 \%$ of the population, consume $41 \%$ of total health-care costs. ${ }^{6}$ In Sweden, elderly patients, who represent a high proportion of those with MCCs, accounted for $45 \%$ of hospital admissions and $50 \%$ of bed-days in 2005 . $^{7}$ 
According to an estimate in 2014, elderly care in Sweden cost USD 12.7 billion (SEK 109.2 billion). ${ }^{8}$ A particularly common and challenging group of patients with MCCs are those with concurrent cardiovascular disease, chronic kidney disease, and diabetes mellitus; hereinafter referred to as HND (Heart, Nephrology, and Diabetes mellitus) patients. ${ }^{9,10}$ These three diseases commonly coexist as they share similar disease mechanisms, and the presence of one contributes to an increased risk for developing the others. They contribute significantly to increased healthcare utilization and a high disease burden, but to what extent has not been well described. There is limited information on health-care utilization patterns for patients with MCCs.

Current health-care systems have evolved primarily from a medical specialist perspective, offering separate care processes for each of the HND diagnoses. The systems are not adapted to patients with MCCs, and lead to fragmentation and high health-care utilization. ${ }^{11,12}$ Inadequate design and lack of care coordination around MCC patients' care processes contribute to inefficiencies in care delivery services. ${ }^{13}$ Recently, multidisciplinary care interventions for combined management of patients with MCCs have been introduced. One example of such multidisciplinary care interventions is integrated care clinics. ${ }^{14,15}$

Integrated care involves multidisciplinary teams that provide person-centered care over the entire cycle of care for a specific condition - inpatient, outpatient, and rehabilitative care as well as patient education, engagement, and follow-up. ${ }^{1,15}$ The multidisciplinary aspect in these settings involves collaboration between professions, i.e. physicians, nurses, and paramedicine staff usually within, but sometimes also between, medical specialties. ${ }^{15}$ For the purpose of this article, we refer to multidisciplinary care as "an approach that seeks to improve the quality of care for individual patients, service users and care providers by ensuring that services are well coordinated around their needs". ${ }^{16}$ In addition, we refer to person-centered care as "a partnership between patient, their relatives, and professionals, in health and elderly care and rehabilitation". ${ }^{17}$

There is mixed evidence on how the multidisciplinary and person-centered care models affect the health-care utilization patterns. ${ }^{4}$ A systematic literature review of comprehensive multidisciplinary care programs for patients with MCCs concluded that there was no definitive evidence available to draw conclusions on how such programs affect health utilization patterns. ${ }^{18} \mathrm{~A}$ recent systematic review and metaanalysis concluded that multidisciplinary care coordination interventions for patients with MCCs appear to have the potential for improving primary and secondary outcomes, including care utilization. ${ }^{19}$ The effects of such care programs are different for each type of model and disease. Further evidence is needed to establish if multidisciplinary care interventions affect health-care utilization patterns in patients with specific disease combinations. One published empirical study explores the effects of multidisciplinary care interventions on care utilization patterns of HND patients, but it measured care utilization through hospital admissions only. $^{20}$

The aims of this study were to describe the characteristics of HND patients and to explore the initial effects of a multidisciplinary and person-centered care on total care utilization.

\section{Materials and Methods Study of the Intervention}

This study reports the initial findings of a randomized controlled trial (RCT; CareHND). The RCT investigates several aspects of a multidisciplinary and person-centered clinic, hereafter referred to as the HND center. ${ }^{10,21}$ Patients who fulfilled the inclusion criteria (established cardiovascular disease, diabetes mellitus type 1 or 2, and established kidney disease) and provided written informed consent were randomized to either traditional standard care or to the HND center.

\section{Context of the Intervention}

The study was conducted at the HND center that was established at a tertiary academic medical hospital, Danderyd University Hospital, in Stockholm, Sweden. The hospital is a large Emergency Hospital, with 540 beds, that provides specialist health services for approximately 650,000 persons as well as conducting research and teaching as an affiliated teaching hospital of Karolinska Institutet. In Sweden, the health-care system including prescription drugs is almost exclusively tax-funded. The responsibility for overall health policy lies with the state, but funding and service provision are the responsibility of the region, in particular, primary and hospital-based care.

Primary health-care services are seen as the foundation of the Swedish health-care systems, but a large part of the care is provided by regional hospitals and hospital-based specialists. Municipalities are legally responsible for care provision and the housing needs of elderly people. These organizational boundaries between these providers create care coordination challenges for patient groups, such as 
HND patients, that are dependent on care provision from the municipality as well as primary- and hospital-based care.

\section{Intervention (HND Center)}

The HND center is the second of its kind in the world, after a similar center at St Paul's hospital Vancouver, Canada. ${ }^{20}$ The goal of the outpatient center is to offer comprehensive care to HND patients in an integrated, multidisciplinary, and person-centered manner to improve care coordination, reduce and replace visits to a variety of doctors and nurses, and within a period of one-year, develop sustainable care management plan that can be handed-off to primary care. Upon the HND center's establishment in 2013, clinical and health systems' researchers were tasked to design a research program to compare clinical outcomes, patient experience and costs of HND center care to that of traditional "siloed" care processes. This evaluation was necessary for estimating the value gained or lost when implementing this type of multidisciplinary and personcentered intervention. ${ }^{21}$

The HND center aims to optimize medical care, reduce unnecessary health-care utilization, and potentially lower costs for HND patients. Patients meet the team through a set of outpatient visits, and a junior consultant (JS) then discuss all cases during twice-weekly conferences with the three senior consultants. ${ }^{15}$ The HND center operates on weekdays during office hours. HND Staff is a team of health-care professionals including the junior consultant and a nurse manager supported by an "undersköterska", an equivalent of an American Licensed Practical Nurse (LPN) or a British NHS Health-Care Assistant. This team is complemented with dieticians, physiotherapists, parttime nurses specialized in heart failure, nephrology and diabetes, and two each of nephrologists, cardiologists and endocrinologists on a rotating schedule. Instead of patients visiting the different specialties at different locations as in traditional care model, the HND center is designed from a patient-centered perspective where patients obtain all necessary treatments at a single location.

\section{Data Collection and Measures}

Data were collected from Stockholm County Council's VAL database. The VAL Database collects data from the electronic health records and patient administrative systems of different health-care providers in Stockholm into a single place for medical billing purposes. For this study, data were obtained for the period of January 2008 to
April 2018 for HND patients recruited to the RCT. This period included 5 years of baseline data ahead of the HND center's establishment, and all data up until data analysis.

Data (linked to personal identification numbers) were obtained for 110 HND patients. All data were de-identified using an encryption code and stored in a secure locker under the supervision of responsible persons. All patients were given a unique ID number that could be used to trace the patient's data.

We collected data on general patient characteristics (age, sex and demographics) and care utilization. Since a patient's degree of morbidity can have a large influence on their care utilization, we collected main-diagnosis data in the form of ICD codes.

In this study, care utilization included all care provided by the Swedish health-care system except for non-licensed health-care providers in nursing home settings or assisted living settings. Measures of care utilization included: 1) unplanned visits including outpatient emergency visits (ED visits) and unplanned inpatient visits, 2) inpatient visits both planned and unplanned, 3) length of stay per admission (LOS), and 4) planned outpatient visits (telephone, total inperson visits, primary care, physician and nurse visits).

\section{Data Analysis}

To study the patterns in care utilization before and after patients were included in the HND intervention, we conducted a descriptive quantitative analysis guided by the RCT design. Data were prepared for the analysis such that each row represented a clinical event, such as a visit, associated with a specific patient, specific date, main diagnostic code, visit type, indication if inpatient or outpatient, if acute or planned, and the length of stay for inpatient visits. Data were cleaned by eliminating missing values. After removing missing values, the data needed to be normalized to a common time scale. The aim was to compare care utilization after patients were randomized, but patients were recruited to the RCT intermittently over a few years. In order to compare the intervention and control arms, the time scales of each patient were normalized to a common scale where time zero represented the start of each patient's exposure to the intervention, represented by the first HND-center-visit. Patients in the control arm did not experience a first-HND visit, so a hypothetical first-HND visit was estimated. As patients in both the intervention and control arms had a set date in which they were recruited to the study, patients in the intervention arm would experience their first-HND visit sometime after recruitment. The hypothetical first-HND visit date 
for the control arm was estimated by adding the mean recruitment-to-first-HND-visit time for the intervention arm to the recruitment date of patients in the control arm. All data entries during the first month of intervention were excluded from the analyses because the change to HND care was associated with several visits to transition patients into HND care processes.

Recruitment to the RCT is ongoing since the HND center's establishment. As a result, many patients were recruited within the past few years, and some within a few months of the analysis. This required that we use a 6-month cut-off period because the number of patients with sufficient data would have been unacceptably low using a higher cut-off period. The cut-off period was defined as the minimum number of days (months) HND patients had to be exposed to the respective intervention and control arms before they could be included in the analysis. To adjust for the late entry, all patients whose first-HND visit were less than 6 months before the end of the data collection period were removed from the dataset. For similar reasons, to adjust the data for mortality, all HND patients who died within less than 6 months of the intervention were also removed from the data set.

The dataset consisted of 234,763 observations and 20 variables (such as personal number, RCT arm, health-care provider type, acute or non-acute, clinic/department, visit date, admission and discharge dates, visit type, diagnosis type, i.e. ICD codes and clinical procedures) for the 110 HND patients. After mortality and late entry adjustments, a total of 77 patients had complete data for 6 months following inclusion in the trial. Of these, 42 patients were in the intervention arm and the remaining 35 patients were in the control arm (Figure 1).

Descriptive statistics were used to analyze patient characteristics including age, gender, and ICD codes. Median diagnosis frequencies, based on ICD codes, were used because such variables are heavily skewed right. From these ICD codes, weighted Charlson Comorbidity Index (CCI) scores for both the intervention and control arms at the baseline were also calculated. ${ }^{22,23}$ The CCI is a method used to categorize comorbidities of patients based on ICD codes, where each category has its associated weight (from 1 to 6 ) based on the adjusted risk of mortality. Weighted CCI scores were calculated in $\mathrm{R}$ package called "Comorbidity" version 0.4.1. The comorbidity score for a patient is the sum of all weights, and the higher the score the more likely mortality as an outcome. ${ }^{22,23}$ Descriptive statistics were also used to present the CCI scores.

For all care utilization variables, the mean per-patient monthly frequency for the baseline period of 24 months prior to normalized time zero was calculated for all patients in the study. The median of the patient means was then calculated, as the distribution of the means was heavily skewed right. Patients with zero values were included in these calculations. However, when calculating the LOS per admission per patient, we did not include patients with zero admissions in that calculation. The same calculations were run for the post-intervention 6-month periods for the intervention and control arms. A Wilcoxon Ranked Sum Test was performed to test if

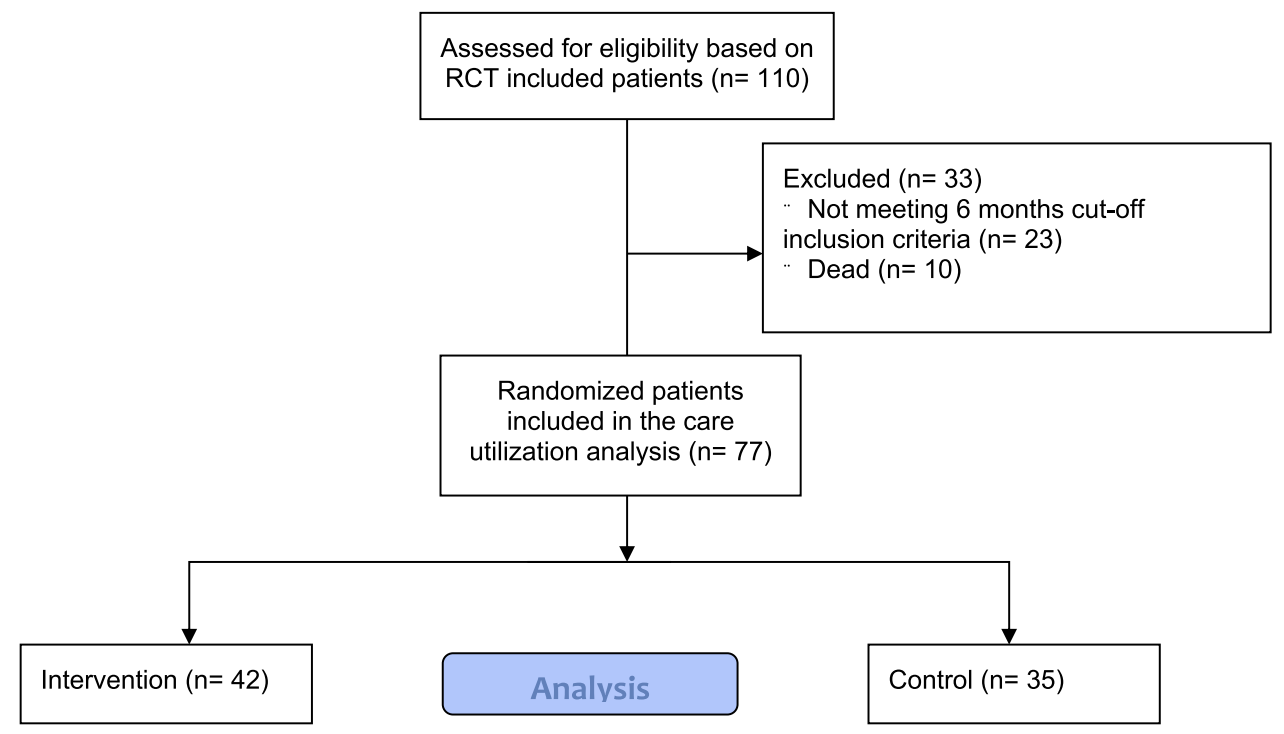

Figure I Flow chart of the patients included in the care utilization analysis. 
there was a significant difference between the intervention and control arms with respect to each care utilization variable.

We also stratified the care utilization by age group, because we anticipated differences.

\section{Ethical Considerations}

The study was approved by the Stockholm Regional Ethics Committee (Diary Numbers 2014/384-31/1 and 2017/99931/2). Data processing was in compliance with ethical approval, and in accordance with the Declaration of Helsinki. Informed consent was obtained from all study participants. Upon request, the authors will share deidentified VAL data, the R script and the post-processing dataset, used in the current study, with other research institutions specifically for research purposes - so long as the data sharing process is in compliance with the General Data Protection Regulation (GDPR) policy. Data will be available for 10 years after publication. The corresponding author can be contacted for data sharing purposes. Further information on Individual Participant Data Sharing are available on clinicaltrials.gov.

\section{Results}

\section{Patient Characteristics}

The distribution of study participants' demographics is shown in Table 1. Both arms contain more men than women, and the mean age was 75.6 years. The majority of patients in both arms were in the age range 70-79 years $-48 \%$ and $46 \%$ for intervention and controls arms, respectively.

Fifty-nine different main diagnoses were identified, and the 20 most frequent diagnoses in each group are presented in Appendix A, with the corresponding frequencies. The most predominant diagnosis was type 2 diabetes mellitus, for which those patients sought health care 523 times during the selected period.

There were differences between the groups in terms of most frequently assigned diagnoses and the presence or absence of certain diagnoses as shown in Appendix A. For instance, proliferative diabetic retinopathy was found only in the intervention arm, while non-proliferative diabetic retinopathy was found in both arms. Similarly, unspecified cerebral infarcts were found only in the control arm and heart valve prostheses were found only in the intervention arm. Other noticeable differences between the two groups were that essential hypertension and angina pectoris were
Table I Baseline Demographics and Multimorbidity of Study Groups (Intervention Compared to Control)

\begin{tabular}{|c|c|c|c|}
\hline Characteristics & & $\begin{array}{l}\text { Control } \\
\text { Arm } \\
\text { (STAN) }\end{array}$ & $\begin{array}{l}\text { Intervention } \\
\text { Arm (HND) }\end{array}$ \\
\hline $\begin{array}{l}\text { Number of } \\
\text { participants }\end{array}$ & & 35 & 42 \\
\hline Gender & $\begin{array}{l}\text { Female } \\
\text { Male }\end{array}$ & $\begin{array}{l}\text { II (3I.4\%) } \\
24(68.6 \%)\end{array}$ & $\begin{array}{l}7(16.7 \%) \\
35(83.3 \%)\end{array}$ \\
\hline $\begin{array}{l}\text { Age years mean } \\
\text { (Median) }\end{array}$ & & $76.26(75)$ & $74.2(75)$ \\
\hline $\begin{array}{l}\text { Participants based on } \\
\text { age subgroups (\%) }\end{array}$ & $\begin{array}{l}50-59 \\
60-69 \\
70-79 \\
80-89\end{array}$ & $\begin{array}{l}0(0 \%) \\
7(20 \%) \\
16(45.7 \%) \\
12(34.3 \%)\end{array}$ & $\begin{array}{l}\text { I (2.38\%) } \\
9(21.4 \%) \\
20(47.6 \%) \\
12(29 \%)\end{array}$ \\
\hline $\begin{array}{l}\text { Median diagnoses per } \\
\text { patient (Range) }\end{array}$ & & $14.0(5-44)$ & $17.0(5-38)$ \\
\hline CCI Score & $\begin{array}{l}\text { Median } \\
\text { Mean }\end{array}$ & $\begin{array}{l}4.0 \\
4.3\end{array}$ & $\begin{array}{l}5.5 \\
5.4\end{array}$ \\
\hline
\end{tabular}

Abbreviation: $\mathrm{CCl}$, Charlson Comorbidity Index.

proportionally more in the control arm (56 and 18) compared to the intervention arm (35 and 12).

The number of multimorbidities ranged from 2 to 44 diagnoses per patient (Table 1). Aggregate CCI scores for the intervention and control arms are shown in Table 1, with the HND patients having scores a full point higher (mean $=5.4)$ than controls $($ mean $=4.3)$.

\section{Initial HND Center Effects on Health-Care Utilization}

The patients are high consumers of care, typically experiencing three to four outpatient visits and one inpatient visit per month, and spending 3 to 5 days in the hospital per admission. This group of 77 patients experiences approximately 250 outpatient visits, and 300 bed days each month. In some cases, these patients were hospitalized for up to 34 days. The median per month care utilization variables at baseline and 6 months after randomization are shown in Table 2. The median LOS in the intervention and control arms were 3.5 and 4.0 days, respectively, compared to 4.0 at baseline. Nurse and telephone visits increased from baseline in the intervention arm to 2.1 and 1.2, respectively, from 2.0 and 1.0. The control arm remained the same, with a slight decrease to 
Table 2 Comparison of Care Utilization Variables for 24 Months Pre- and 6 Months Post Randomization

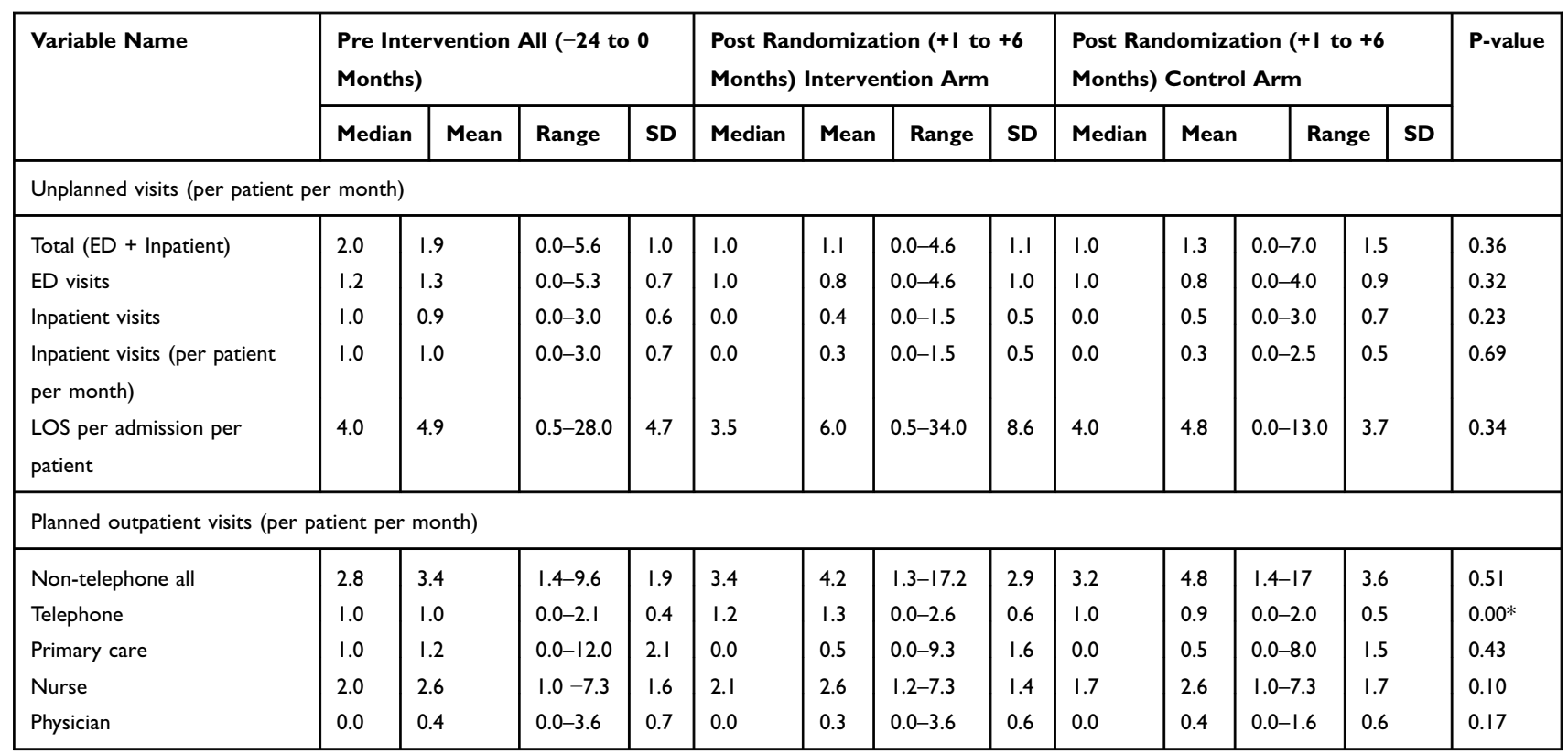

Note: *statistically significant.

Abbreviations: ED, Emergency Department; LOS, Length of Stay.

1.7 nurse visits. While the medians remained the same in both arms, the mean physician visits slightly decreased in the intervention arm. The remaining care utilization variables decreased by the same amount from baseline in both arms. The only statistically significant difference between the two arms was seen in telephone visit frequencies.

Further exploring care utilization with a sub-group analysis by age shows that there are an equal number of diagnoses across the 60-69 age group, and the 80-89 age group, but the number of visits in these groups is different in the two arms (Table 3).

\section{Discussion}

The 77 patients included in this study presented a large variation in terms of number of diagnoses, and the patients in the intervention arm appeared to be sicker than those in the control arm, as indicated by the CCI score which was a point higher in the intervention arm. The condition of these patients is care intensive, with anywhere from five to 44 diagnoses per patient that demand several visits and a few hundred bed days each month. Within this care utilization analysis, the initial effects of the HND center did not result in a clear decrease or increase in care utilization. There was a statistically significant increase in telephone consults in the intervention arm. There was a non-statistically significant decrease in the median LOS per admission and an increase in nurse visits in the intervention arm.
The increase in telephone visits and nurse visits aligns with the intention of the HND center, which aimed to replace, when appropriate, physician visits with nurse visits, and in-person visits with telephone consults. A consequent reduction in physician and in-person visits may manifest in the long term which was also observed from the small decrease in the mean physician visits per patient per month in our findings. The HND RCT is ongoing, and a better estimate of the difference in care utilization will emerge as more patients are recruited. The increase in telephone consultations could be related to managing patients' transitions from uncoordinated care into integrated care.

Overall, there was no substantial increase in care utilization in the intervention arm, despite the fact that these patients were sicker, with a median and mean CCI score both a full point higher. Patients in the intervention arm experienced a median of three more diagnoses than those in the control arm. While the ages of the two groups were similar, there were more males in the intervention arm compared to the control arm $(83.3 \%$ vs $68.6 \%)$, and males tend to consume more health care. ${ }^{24-26}$

Our findings corroborate the difficulty of obtaining statistically significant findings from studies evaluating a multidisciplinary intervention for MCC patients. ${ }^{4}$ A recent Cochrane systematic review studying the effectiveness of health-service or patient-oriented interventions in people with MCCs found that there were no clear positive 
Table 3 Distribution of Median Visits per Patient and Median Number of Diagnoses per Age Group

\begin{tabular}{|c|c|c|c|c|}
\hline & \multicolumn{2}{|l|}{ Intervention Arm $(n=42)$} & \multicolumn{2}{|l|}{ Control Arm $(n=35)$} \\
\hline & $\begin{array}{l}\text { Median Overall (Includes All } \\
\text { Types) Visits per Patient (Range) }\end{array}$ & $\begin{array}{l}\text { Median No of } \\
\text { Diagnoses per Patient } \\
\text { (Range) }\end{array}$ & $\begin{array}{l}\text { Median Overall (Includes All } \\
\text { Types) Visits per Patient (Range) }\end{array}$ & $\begin{array}{l}\text { Median No of } \\
\text { Diagnoses per Patient } \\
\text { (Range) }\end{array}$ \\
\hline \multicolumn{5}{|l|}{ Age Group (years) } \\
\hline $50-59$ & $9.0(\mathrm{NA})(\mathrm{n}=\mathrm{I})$ & $14.0(\mathrm{NA})(\mathrm{n}=\mathrm{I})$ & $N A(n=0)$ & $N A(n=0)$ \\
\hline $60-69$ & $12.0(1-257)(n=9)$ & $8.0(4-12)(n=9)$ & $1.50(|3-6|)(n=7)$ & $8.0(6-10)(n=7)$ \\
\hline 70-79 & $9.0(I-522)(n=20)$ & $13.0(5-21)(n=20)$ & $6.0(6-110)(n=16)$ & $9.0(3-19)(n=16)$ \\
\hline $80-89$ & $4.50(1-350)(n=12)$ & $12.0(6-30)(n=12)$ & $9.0(7-62)(n=12)$ & $14.0(7-37)(n=12)$ \\
\hline
\end{tabular}

improvements in clinical outcomes, health-care utilization and costs, and medication adherence. ${ }^{4}$ However, there is support in the literature that the key components in the HND clinic's design, i.e. integrated, multidisciplinary, interprofessional collaboration incorporating a patient-centered perspective could have a positive overall effect on patient satisfaction and quality of care. ${ }^{27-29}$ In fact, another study performed around the HND center found that health-care professionals working at the center were confident that this is the optimal approach for treating these patients. ${ }^{30}$ It could also be beneficial that patients receive most of their care at a single location from a single provider team. ${ }^{31}$

These patients are complex both from clinical and managerial perspectives. From the clinical perspective, it is challenging to stratify these patients into groups based on the distinctive nature of their medical conditions in order to better understand and improve care delivery. These major differences could be influenced if physicians in the intervention arm used different ICD codes to classify their patient visits, compared to physicians in the control arm. These kinds of inconsistencies and a lack of established standards render scarce health-care resources wasted, compromising patient care and leading to poorer patient outcomes. ${ }^{32-34}$

The high magnitude and variation of both diagnoses and care utilization are evidence that maintaining homogeneity in randomized controlled trials conducted on HND patients, and patients with MCCs, in general, is challenging. The underlying complexity and heterogeneity of HND patients in both arms were evident by the CCI scores, and when grouped by age. Different disease progression patterns were observed in the age sub-group visit frequencies (Table 3) and number of diagnoses (Appendix A). Perhaps a deeper analysis with new approaches such as cluster networking and machine learning could be applied to explore clinically relevant sub-groups of patients with MCCs. In a separate study, we were able to identify three distinct sub-groups of HND patients, i.e. HND patients with chronic obstructive pulmonary disease, kidney transplant, and paroxysmal ventricular tachycardia by applying machine learning algorithms. ${ }^{35}$ These methods could be incorporated in the design and randomization of controlled trials such that the heterogeneity and bias in the intervention and control groups are reduced. Innovative use of statistics and machine learning in the randomized controlled trials could generate a new ecosystem for validation and exploration that could complement or replace these kinds of studies. ${ }^{36,37}$ The approach to identify sub-samples of subjects for controlled trials may have better results in demonstrating the effects of interventions. The identification of sub-groups, specific disease combinations and clusters of diseases is likely to provide useful insights during the management of patients with MCCs. ${ }^{38}$ We intend to incorporate the insights and data of this study in the design and validation of a predictive decision support model for HND patients.

\section{Limitations}

The sample size of the study is relatively small and the HND patients are all from a single hospital. Therefore, generalizability of the findings may be limited. For the primary analysis, we only used the major diagnoses listed in the data sets and did not include other diagnoses. Further, to fully understand the effects of this intervention, care utilization should be evaluated together with other outcomes and costs. The effects on clinical outcomes and patient experience will be reported upon completion of the RCT.

\section{Conclusion}

HND patients consume large amounts of health-care resources including high hospitalization rates, emergency department visits and frequent encounters with professionals in the health-care system. Providers are left with the task of 
delivering this magnitude of care to treat a diverse set of 59 separate diagnoses. The complexity of this situation captures the challenges faced by providers implementing integrated care interventions, and by researchers evaluating such interventions. However, initiatives like the HND center may positively influence health-care utilization in HND patients. Innovative methods like machine learning should be explored in studies designed to evaluate the impact of integrated care on care utilization of patients with multiple chronic conditions.

\section{Acknowledgments}

This work was financially supported by Vårdalstiftelsen, with additional financial support from FORTE, the Kamprad Family Foundation, and Strategic Research Area Health Care Science. PM was funded by the Strategic Research Area Health Care Science, Karolinska Institutet/Umeå University during the project period. GK was financially supported by KID funding by Karolinska Institutet. The funders were not involved in the study design; in the collection, analysis and interpretation of the data; in the writing of the report; nor in the decision to submit the paper for publication.

\section{Author Contributions}

All authors contributed to data analysis, drafting or revising the article, gave final approval of the version to be published, and agree to be accountable for all aspects of the work.

\section{Disclosure}

One of the co-authors, JS, is the manager of the HND center. JS reports grants from Kamprad Foundation, grants from Stockholm County, during the conduct of the study; and has received speaker honoraria from GENZYME, AbbVie, AstraZeneca, Boehringer, and Bristol-Myers Squibb. The authors report no other conflicts of interest in this work.

\section{References}

1. Levin, Levin A, Chaudhry MR, et al. Diabetes, kidney disease and cardiovascular disease patients. Assessing care of complex patients using outpatient testing and visits: additional metrics by which to evaluate health care system functioning. Nephrol Dial Transplant. 2009;24(9):2714-2720. doi:10.1093/ndt/gfp180

2. Hopman P, Heins MJ, Korevaar JC, Rijken M, Schellevis FG. Health care utilization of patients with multiple chronic diseases in the Netherlands: differences and underlying factors. Eur J Intern Med. 2016;35:44-50. doi:10.1016/j.ejim.2016.08.025

3. Mercer S, Furler J, Moffat K, Fischbacher-Smith D, Sanci L. Multimorbidity: Technical Series on Safer Primary Care. Geneva: World Health Organization;2016. Licence: CC BY-NC-SA 3.0 IGO. Available from: https://apps.who.int/iris/bitstream/handle/10665/252275/ 9789241511650-eng.pdf?sequence=1. Accessed December 6, 2019.
4. Smith SM, Wallace E, O'Dowd T, Fortin M. Interventions for improving outcomes in patients with multimorbidity in primary care and community settings (Review). Cochrane Database Syst Rev. 2017;(3). doi:10.1002/14651858.CD006560.pub3.www.cochranelibrary.com

5. Stanton MW. The high concentration of U.S. Health care expenditures. Health Care (Don Mills). 2006;(19).

6. Buttorff C, Ruder T, Bauman M. Multiple Chronic Conditions in the United States. Santa Monica, CA: RAND Corporation; 2017.

7. Socialstyrelsen. National Board of Health and Welfare (Socialstyrelsen) (2001) Health and Medical Statistics Yearbook 2000 (Hälso-Och Sjukvårdsstatistisk Arsbok 2000). Stockholm, Sweden: The National Board of Health and Welfare; 2007.

8. Swedish Care International. Available from: https://sci.se/elderly-care -in-sweden/. Accessed December 6, 2019. Published 2019.

9. Suckling R, Gallagher H. Chronic kidney disease, diabetes mellitus and cardiovascular disease: risks and commonalities. J Ren Care. 2012;38:4-11. doi:10.1111/j.1755-6686.2012.00274.x

10. Spaak J. Challenging complex diseases. In: Goldsmith D, Covic A, Spaak J, editors. Cardio-Renal Clinical Challenges. Cham, Heidelberg, New York, Dordrecht, London: Springer; 2015. doi:10.1007/978-3-319-09162-4

11. Stange KC. The Problem of Fragmentation and the THE PROBLEM of FRAGMENTATION. 2009:100-103. doi:10.1370/afm.971.AN

12. Clarfield AM, Bergman H, Kane R. Fragmentation of care for frail older people - an international problem. Experience from three countries. J Am Geriatrics Soc. 2001;49(December 1998):1714-1721.

13. Wolff JL, Starfield B, Anderson G. Prevalence, expenditures, and complications of multiple chronic conditions in the elderly. Arch Intern Med. 2002;162(20):2269-2276. doi:10.1001/archinte.162.20.2269

14. WHO Regional Office for Europe. Integrated Care Models: An Overview World Health Organization Regional Office for Europe; 2016.

15. Spaak J. Novel combined management approaches to patients with diabetes, chronic kidney disease and cardiovascular disease. J R Coll Physicians. 2017;47(1):83-87. doi:10.4997/JrcPe.2017. 118

16. Goodwin N, Smith J, Davies A, et al. Integrated care for patients and populations: improving outcomes by working together. In: A Report to the Department of Health and the NHS Future Forum. Kings Fund Nuff Trust; 2012:1-20.

17. GPCC. CENTRE for PERSON-CENTRED CARE. University of Gothenburg. Available from: https://gpcc.gu.se/english/about-gpcc /person — centred-care. Published 2019.

18. De BSR, Versnel N, Lemmens LC, et al. Comprehensive care programs for patients with multiple chronic conditions: a systematic literature review. Health policy. 2012;107:108-145. doi:10.1016/j. healthpol.2012.06.006

19. Kastner M, Cardoso R, Mn YL, et al. Effectiveness of interventions for managing multiple high-burden chronic diseases in older adults: a systematic review and meta-analysis. CMAJ. 2018;190(34). doi:10.1503/cmaj.171391

20. Weber C, Beaulieu M, Djurdjev O, et al. Towards rational approaches of health care utilization in complex patients: an exploratory randomized trial comparing a novel combined clinic to multiple specialty clinics in patients with renal disease-cardiovascular disease-diabetes. Nephrol Dial Transplant. 2012;27(suppl 3):iii104-iii110. doi:10.1093/ ndt/gfr292

21. NCT03362983. Integrated, multidisciplinary, person-centered care for patients with complex comorbidities: heart, kidney and diabetes (CareHND). Available from: https://www.clinicaltrials.gov/ct2/show/ NCT03362983?term $=03362983 \&$ rank=1. Accessed December 6, 2019. Published 2019.

22. Charlson ME, Charlson ME, Pompei P, et al. A new method of classifying prognostic comorbidity in longitudinal studies: development and validation. J Chronic Dis. 1987;40(5):373-383. doi:10.1016/ 0021-9681(87)90171-8 
23. Charlson ME, Charlson ME, Charlson RE, et al. The Charlson comorbidity index is adapted to predict costs of chronic disease in primary care patients. J Clin Epidemiol. 2008;61(12):1234-1240. doi:10.1016/j.jclinepi.2008.01.006

24. Stone PH, Thompson B, Anderson HV, et al. Influence of race, sex, and age on management of unstable angina and non-Q-wave myocardial infarction: the TIMI III registry. JAMA. 1996;275(14):1104-1112.

25. Correa-de-Araujo R, Stevens B, Moy E, Nilasena D, Chesley F, McDermott K. Gender differences across racial and ethnic groups in the quality of care for acute myocardial infarction and heart failure associated with comorbidities. Women's Heal Issues. 2006;16 (2):44-55. doi:10.1016/j.whi.2005.04.003

26. Hollenbeak CS, Weisman CS, Rossi M, Ettinger SM. Gender disparities in percutaneous coronary interventions for acute myocardial infarction in Pennsylvania. Med Care. 2006;44(1):24-30. doi:10.1097/01.mlr.0000 188915.66942.69

27. Epstein NE. Multidisciplinary in - hospital teams improve patient outcomes: a review. Surg Neurol Int. 2014;5:295-304. doi:10.4103/ 2152-7806.139612

28. Väre P, Nikiphorou E, Hannonen P, Sokka T. Delivering a one-stop, integrated, and patient-centered service for patients with rheumatic diseases. SAGE Open Med. 2016;4:205031211665440. doi:10.1177/ 2050312116654404

29. Mastellos N, Care P, Health P, Health P. Integrated care case assessing patients' experience of integrated care: a survey of. 2014;14(June):1-9.

30. Evén G, Spaak J, von Arbin M, et al. Health care professionals' experiences and enactment of person-centered care at a multidisciplinary outpatient specialty clinic. J Multidiscip Heal. 2019;12(12):137-148. doi:10.2147/JMDH.S186388

31. Tyler KH, Haverkos BM, Hastings J, et al. The role of an integrated multidisciplinary clinic in the management of patients with cutaneous lymphoma. Front Oncol. 2015;5(June):1-5. DOI:10.3389/fonc.2015.00136
32. Jakovljević M, Ostojić L. Comorbidity and multimorbidity in medicine today: challenges and opportunities for bringing separated branches of medicine closer to each other. Psychiatr Danub. 2013;25 Suppl 1(1):18-28.

33. Fortin M, Lapointe L, Hudon C, Vanasse A. Multimorbidity is common to family practice: is it commonly researched? Can Fam Physician. 2005;51:244-245.

34. Marengoni A, Angleman S, Melis R, et al. Aging with multimorbidity: a systematic review of the literature. Ageing Res Rev. 2011;10 (4):430-439. doi:10.1016/j.arr.2011.03.003

35. Rafiq, Keel G, Mazzocato P, Spaak J, Savage C, Guttmann C. Deep Learning Architectures for Vector Representations of Patients and Exploring Predictors of 30-Day Hospital Readmissions in Patients with Multiple Chronic Conditions. Vol. 1. Springer International Publishing; 2019. doi:10.1007/978-3-030-12738-1

36. Poucke S, Thomeer M, Heath J, Vukicevic M. Are randomized controlled trials the $(\mathrm{G})$ old standard? From clinical intelligence to prescriptive analytics. Int J Internet Res. 2016;18(7):e18.

37. Holmes CC, Watson JA. Machine learning for randomised controlled trials: identifying treatment effect heterogeneity with strict control of type I error. bioRxiv. 2018;1-12. doi:10.1101/330 795

38. Hussain, Hussain MA, Katzenellenbogen JM, et al. Complexity in disease management: a linked data analysis of multimorbidity in Aboriginal and non-Aboriginal patients hospitalised with atherothrombotic disease in Western Australia. PLoS One. 2018;13(8): e0201496. doi:10.1371/journal.pone.0201496
Journal of Multidisciplinary Healthcare

\section{Publish your work in this journal}

The Journal of Multidisciplinary Healthcare is an international, peerreviewed open-access journal that aims to represent and publish research in healthcare areas delivered by practitioners of different disciplines. This includes studies and reviews conducted by multidisciplinary teams as well as research which evaluates the results or conduct of such teams or healthcare processes in general. The journal covers a very wide range of areas and welcomes submissions from practitioners at all levels, from all over the world. The manuscript management system is completely online and includes a very quick and fair peer-review system. Visit http://www.dovepress.com/testimonials. php to read real quotes from published authors. 